\title{
Des maladies chroniques et des Ferrari
}

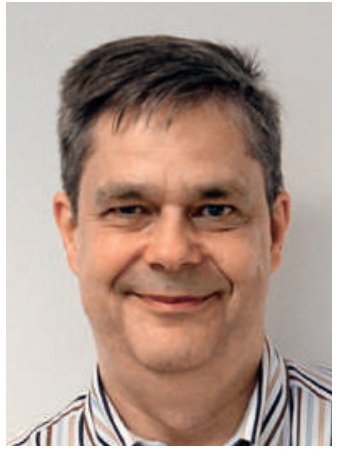

Eberhard Wolff
* PD Dr ès sc. soc.

Eberhard Wolff est licencié en études culturelles, historien de la médecine et membre de la rédaction Histoire de la médecine du Bulletin des médecins suisses.
Le patient (ou la patiente) arrive au cabinet et énumère ses maux. Le médecin écoute, pose des questions, établit un diagnostic, délivre une ordonnance. $\mathrm{Au}$ suivant! Ce qui est souvent décrié comme l'effrayant portrait d'une médecine à la chaîne peut s'avérer très reposant pour les deux parties - quand on le compare à son opposé, symptômes diffus et diagnostic difficile. Et même lorsque ceux-ci sont à peu près clairs, une thérapie médicamenteuse peut être inutile ou ne pas suffire. Le patient insatisfait ne cesse alors de revenir, le traitement dure des années... Ce genre de situation est souvent le résultat d'une maladie chronique.

Les débats publics visent à vulgariser les thèmes abordés et parfois, même une discussion embrouillée peut aider à y voir clair - par exemple, lorsqu'elle démontre la complexité d'un sujet. C'est ce qui s'est passé récemment autour des «maladies chroniques» lors d'un débat organisé par l'Académie Paulus et la Fondation Careum sur le thème de la «formation du patient» [1].

Les maladies chroniques peuvent représenter un immense défi pour toutes les parties concernées. Elles exigent souvent une approche à la fois spécifique et lourde. Roberto Brioschi, directeur du Centre de traitement de la douleur de la Clinique de réadaptation de Bad Zurzach, essaie d'établir en consultation une relation avec le patient et de comprendre le «modèle» mental qu'il a de sa maladie. Lorsqu'il s'agit du squelette, il lui arrive d'employer la métaphore de la Ferrari pour décrire le patient.

De la Ferrari au siège arrière d'une Vespa: Anna Sax, économiste de la santé et conseillère de fondation d'Info-Entraide Suisse, explique que dans les cas de maladie chronique, les médecins décrivent leur rôle comme celui d'un passager accompagnant leur patient à travers la maladie. Selon Jörg Haslbeck, responsable de la formation des patients chez Careum, la santé est alors "coproduite» par les spécialistes, le patient et l'environnement. Bien sûr, comme l'explique l'éthicienne clinique Tanja Krones de Zurich, certains patients veulent être guidés par les médecins. Mais au moins un patient sur deux veut participer avec ceux-ci aux décisions concernant sa santé. Et il arrive qu'un non-médecin ou un autre patient fasse mieux passer l'information - «d'égal à égal».

Les maladies chroniques sont éprouvantes, surtout pour les patients. Car changer de comportement est difficile - et il faut prendre en compte la vie quotidienne. Une maladie chronique exige du patient une implication plus active et des connaissances beaucoup plus étendues. D'où l'idée de la «formation des patients».
Un patient informé peut s'exprimer, comprendre, décider en toute conscience. Cela lui donne la sensation de pouvoir agir sur son destin, d'être responsable. Les groupes d'entraide ne sont qu'un lieu de formation des patients parmi d'autres: Internet joue en la matière un rôle croissant.

Ce qui est en jeu dépasse l'information factuelle et la «compliance»: la formation des patients vise aussi leurs comportements, leurs attitudes. Il s'agit de soutenir leur motivation, leur activité propre et leur participation pour les rendre plus sûrs d'eux et plus autonomes, afin de les prémunir contre un glissement vers l'apathie ou la dépression [2].

Lorsqu'elle prend la forme d'un DVD pédagogique, comme il est fréquent aux Etats-Unis, la formation des patients est peu efficace; l'entretien personnel permet au patient de mieux comprendre les problèmes.

De cette discussion animée, je tire le bilan suivant: la formation des patients est aujourd'hui une sorte de «panacée» très en vogue. Mais il en va comme de toutes les «panacées»: pour sensée qu'elle paraisse de prime abord, il convient de l'appréhender avec une bonne dose de scepticisme, comme les intervenants en ont fait preuve à plusieurs reprises. Exige-t-on trop de la «formation des patients»? N'est-elle - d'un côté que l'exercice d'un contrôle indirect, voire une manipulation? Impose-t-elle aux patients le corset étouffant d'une responsabilité trop lourde et d'un contrôle de soi permanent? Ne sert-elle qu'à faire des économies à bon compte? Ou génère-t-elle - de l'autre côté des patients méfiants, zappant d'un médecin à l'autre (Krones), nombrilistes, désorientés ou capricieux, et cela est-il mauvais?[3] Qu'est-ce qu'une «bonne» formation des patients? Et comme le demandait l'animatrice du débat, Susanne Brauer de l'Académie Paulus: qu'en est-il de ceux qui ne veulent pas être formés? Le patient a-t-il un droit de refus?

Même si une telle «panacée» existe, il convient donc de l'employer avec circonspection.

Eberhard Wolff*

1 Expertentum in eigener Sache. Mehr Gesundheit durch Patientenbildung? Débat public du 17 avril 2013 au Volkshaus Zurich.

2 Lorig K et al. Gesund und aktiv mit chronischer Krankheit leben (Editeurs Jörg Haslbeck et Ilona Kickbusch). Zurich: Careum; 2013 (2e éd. corrigée).

3 Sax A. Interview de Louis Litschgi, médecin de famille. Care Management. 2012; 5 (5/6): 11-2.

Litschgi L. Der Arzt auf dem Sozius. Tolerieren statt dirigieren. PrimaryCare. 2012; 12 (1): 8-10. 